\title{
Myelin and lymphocyte protein 2 regulates cell proliferation and metastasis through the Notch pathway in prostate adenocarcinoma
}

\author{
Chenglong Zheng ${ }^{1,2 \#}$, Ji Wang ${ }^{1 \#}$, Jian Zhang ${ }^{3}$, Shujuan Hou ${ }^{1}$, Yanfei Zheng ${ }^{1}$, Qi Wang ${ }^{1}$ \\ ${ }^{1}$ National Institute of Traditional Chinese Medicine (TCM) Constitution and Preventive Medicine, Beijing University of TCM, Beijing, China; \\ ${ }^{2}$ Department of Andrology, Beijing Gulou Hospital of TCM, Beijing, China; ${ }^{3}$ Department of Preventive Treatment, Beijing Hospital of TCM, \\ Capital Medical University, Beijing, China \\ Contributions: (I) Conception and design: C Zheng, J Zhang, Y Zheng; (II) Administrative support: Y Zheng, Q Wang; (III) Provision of study \\ materials or patients: C Zheng, Y Zheng; (IV) Collection and assembly of data: J Zhang, J Wang, S Hou; (V) Data analysis and interpretation: C \\ Zheng, J Zhang, Y Zheng, J Wang; (VI) Manuscript writing: All authors; (VII) Final approval of manuscript: All authors. \\ \#These authors contributed equally to this work. \\ Correspondence to: Qi Wang. Beijing University of TCM, Bei San Huan East Road, Chaoyang District, Beijing 100029, China. \\ Email: wangvxmyczh796@163.com; Yanfei Zheng. Beijing University of TCM, Bei San Huan East Road, Chaoyang District, Beijing 100029, China. \\ Email: loupkan5918@163.com.
}

Background: Myelin and lymphocyte protein 2 (MAL2) is a proven oncogene in some human tumors. However, currently, little is known about the function of MAL2 in prostate adenocarcinoma (PRAD). This study sought to investigate the role of MAL2 on PRAD progression.

Methods: MAL2 expression in PRAD was first analyzed by the Gene Expression Profiling Interactive Analysis (GEPIA) database. The reverse transcription-quantitative polymerase chain reaction (RT-qPCR) assay and Western blot assay were used to detect the expression of MAL2 in PRAD tissues and cell lines. Additionally, immunohistochemistry (IHC) straining was used to detect the expression of MAL2 in PRAD pathological tissues. The Cell Counting Kit-8 (CCK-8) assay, clone formation assay and Flow cytometry were performed to investigate the effect of MAL2 on PRAD cell proliferation and cell apoptosis. Cell migration and invasion were measured by Transwell assay. The effect of MAL2 on epithelial-mesenchymal transition (EMT) progression and the Notch signaling pathway in PRAD was also investigated.

Results: MAL2 was discovered to be obviously upregulated in PRAD tissues and cell lines. The upregulation of MAL2 was closely associated with tumor, nodes and metastases (TNM) stage, the Gleason score and metastasis of PRAD patients, and affected the prognosis of PRAD patients. Functionally, the depletion of MAL2 suppressed cell proliferation, migration, invasion, and EMT progression, and promoted cell apoptosis of PRAD cells. In an in vivo experiment, MAL2 knockdown significantly suppressed tumor growth in mice. Further, inhibiting the Notch pathway reversed the effect of MAL2 knockdown on PRAD progression.

Conclusions: In sum, MAL2 was found to be upregulated in PRAD, and appears to act as a carcinogen in PRAD. Additionally, MAL2 appears to regulate PRAD progression through the Notch signaling pathway.

Keywords: Myelin and lymphocyte protein 2 (MAL2); Notch pathway; prostate adenocarcinoma (PRAD)

Submitted Feb 25, 2021. Accepted for publication Apr 28, 2021.

doi: $10.21037 /$ tau-21-244

View this article at: http://dx.doi.org/10.21037/tau-21-244 


\section{Introduction}

Prostate adenocarcinoma (PRAD) is one of the most common cancers in males, and has the highest incidence of tumors in the urinary system. In the United States, PRAD has the highest incidence of all cancers, and the mortality rate of PRAD is second only to lung cancer (1). The incidence of PRAD in Asian countries, such as China, Japan, and India, is much lower than that in European and American countries, but the incidence of PRAD has been increasing in recent years (2). According to Global Cancer Statistics, in 2018, there were an estimated 1.3 million new cases of PRAD, and 359,000 related deaths worldwide (3). Current treatment methods include surgery, radiation therapy, chemotherapy, immunotherapy, and hormone therapy (4). Multiple tumor markers have been reported to be associated with prognosis in PRAD patients, such as Ki67, p53, p16, and phosphatase and tensin homolog deleted on chromosome ten (PTEN) (5). However, these markers have not been validated in prospective studies, and are not part of the routine examination of PRAD patients. Thus, it is very important to study effective drug targets for PRAD treatment.

Myelin and lymphocyte protein 2 (MAL2) is a 4-time transmembrane protein composed of 176 amino acid residues, which belongs to the MAL proteolipid family. MAL2 is mainly involved in endocytosis, and mediates material transport between cells under physiological conditions (6,7). Endocytosis is an intracellular transport pathway used to transport membrane-binding proteins and exogenous goods from the basolateral to the apical surface. In epithelial cells, MAL2 affects the function of ordered membrane domains to modulate proinflammatory cell signaling (8). Studies have shown that the expression of MAL2 in hepatoma cells is positive, and is directly related to the endocytosis of hepatoma cells (9). Additionally, it has been reported that the expression of MAL2 increases dramatically in pancreatic cancer tissues, and is closely related to the prognosis of pancreatic cancer patients (10). These findings suggest that MAL2 is an important molecule involved in tumorigenesis and malignant progression. However, the role of MAL2 in PRAD had not yet been specifically defined.

In this study, a reverse transcription-quantitative polymerase chain reaction (RT-qPCR) assay and a Western blot assay were used to detect the expression of MAL2 in PRAD tissues and cells. The correlations between MAL2 expression and the clinical parameters of PRAD patients were also analyzed. The effect of MAL2 knockdown on cell proliferation, migration, invasion, and the epithelialmesenchymal transition (EMT) progression of PRAD cells was also explored. Finally, the effect of MAL2 on the Notch pathway in the progression of PRAD was also studied. We present the following article in accordance with the MDAR and ARRIVE reporting checklist (available at http://dx.doi. org/10.21037/tau-21-244).

\section{Methods}

\section{Clinical tissue samples}

A total of 58 surgical specimens were collected from PRAD patients undergoing resection at the Beijing University of Traditional Chinese Medicine. All patients had a definite diagnosis of PRAD by transrectal prostate biopsy before surgery. No patient received any adjuvant therapy of endocrine, radiotherapy, or chemotherapy before surgery. All patients consented to their preoperative samples being examined in this study. The samples were placed in the cryopreservation tube in liquid nitrogen, and then stored in a cryopreservation freezer. All procedures performed in this study involving human participants were in accordance with the Declaration of Helsinki (as revised in 2013). The study was approved by the Ethics Committee of the Beijing University of Traditional Chinese Medicine (NO: 2019028) and informed consent was taken from all the patients.

\section{Cell culture}

Human normal prostate epithelial cells RWPE-1, and PRAD cell lines (DU145, PC3, LNCaP, and C4-2) were purchased from American Type Culture Collection (USA). RWPE-1 cells were cultured in Dulbecco's Modified Eagle Medium (DMEM) medium, which contained 10\% Fetal Bovine Serum (FBS), $5 \mathrm{ng} / \mathrm{mL}$ epidermal growth factor (EGF), $0.05 \mathrm{mg} / \mathrm{mL}$ Bovine Pituitary Extract (BPE), $100 \mathrm{mg} / \mathrm{mL}$ streptomycin, and $100 \mathrm{U} / \mathrm{mL}$ penicillin. PRAD cells were cultured in a Roswell Park Memorial Institute 1640 (RPMI-1640) medium with $10 \%$ FBS, $100 \mathrm{mg} / \mathrm{mL}$ streptomycin, and $100 \mathrm{U} / \mathrm{mL}$ penicillin. All cells were incubated at $37{ }^{\circ} \mathrm{C}$ in an incubator containing $5 \%$ carbon dioxide $\left(\mathrm{CO}_{2}\right)$.

\section{Lentivirus transfection}

Lymph node carcinoma of the prostate (LNCaP) cells and 
C4-2 cells in the logarithmic growth phase were inoculated into 24-well plates with a cell concentration of $1 \times 10^{5}$ cells/ well. We packaged si-MAL2 (MAL2 siRNA forward: 5'-GCAUGAUUUGCAUUGCAAUTT-3' and reverse: 5'-AUUGCAAUGCAAAUCAUGCTT-3') as lentivirus for our experiment. According to the instructions of liposome reagents, si-MAL2 or normal control siRNA (si-NC) was transfected into $\mathrm{LNCaP}$ cells and C4-2 cells. Stable cell lines were established after treatment with $5 \mu \mathrm{g} / \mathrm{mL}$ of puromycin for 2 weeks.

\section{$R T-q P C R$ assay}

Total ribonucleic acid was extracted from PRAD tissues or cell lines using a Trizol reagent (Invitrogen). Next, complementary deoxyribonucleic acid (cDNA) was synthesized by reverse transcription. The cDNA was then used as the amplification template, and the amplification was performed by 7500 qPCR in accordance with the instructions provided for the SYBR Premix EX Taq ${ }^{\mathrm{TM}}$ II kit. The circulation threshold $(\mathrm{Ct})$ of each sample was detected, and the expression of MAL2 was calculated using the $2^{-\Delta \Delta C t}$ method. Glyceraldehyde-3-Phosphate Dehydrogenase was used as the internal reference of MAL2. The primer sequences were as followed: MAL2 forward: 5'-ATGTCGGCCGGCGGAG-3' and reverse: 5'-TTACGGTCGCCATCTTCGCA-3'; $\beta$-actin forward: 5'-GTGCGGCGATTTCATCTTCC-3', and reverse: 5'-CTGCGGCTGAGTTAACAGGA-3'. All primer sequences were designed and synthesized by Guangzhou Ruibo Biological Company (Guangzhou, China).

\section{Western blot assay}

After the cells were fully lysed with a radioimmunoprecipitation assay (RIPA) lysis buffer, the supernatant protein solution was collected by centrifugation at a low temperature and high speed. After determining the protein concentration with a bicinchoninic acid assay (BCA) kit, $20 \mu \mathrm{L}$ of protein solution was electrophoresed on $10 \%$ sodium dodecyl sulphate-polyacrylamide gel electrophoresis gel. The protein isolates were then transferred onto polyvinylidene fluoride (PVDF) membrane using the wet transfer method. The PVDF membrane was sealed with $5 \%$ skimmed milk powder at room temperature for 1 hour. After being fully rinsed with tris-buffered saline (TBST) solution, the primary antibody was added and the membrane was incubated overnight in a shaking bed at $4{ }^{\circ} \mathrm{C}$. Next, the secondary antibody was added, and the membrane was placed in a shaker at room temperature for $1 \mathrm{~h}$. After washing 3 times with TBST, the strips were placed in the enhanced chemiluminescence (ECL) mixture, and then exposed for analysis.

\section{IHC staining}

Biopsy specimens of PRAD were fixed with $4 \%$ formaldehyde solution and embedded in paraffin. Paraffin sections were dewaxed with xylene and hydrated with gradient alcohol and distilled water. Three percent hydrogen peroxide $\left(\mathrm{H}_{2} \mathrm{O}_{2}\right)$ was added, and the sections were left to stand at room temperature for $20 \mathrm{~min}$. Sections were rinsed with $0.01 \mathrm{M} \mathrm{PBS}$ ( $\mathrm{pH}$ 7.4), and a primary antibody was then added at $37^{\circ} \mathrm{C}$ for $40 \mathrm{~min}$. A secondary antibody was added and incubated at $37^{\circ} \mathrm{C}$ for another $40 \mathrm{~min}$, then washed with PBS for 3 times. After adding $0.03 \%$ $\mathrm{H}_{2} \mathrm{O}_{2}-3,3$ '-diaminobenzidine (DAB) solution for $20 \mathrm{~min}$, hematoxylin was redyed for $2 \mathrm{~min}$.

\section{CCK-8 assay}

The transfected LNCaP cells and C4-2 cells were resuspended, and then adjusted to $5 \times 10^{4}$ cells $/ \mathrm{mL}$. One hundred $\mu \mathrm{L}$ cell suspension was inoculated into a 96-well plate. After 8, 24, 48, and $72 \mathrm{~h}$ of inoculation, $10 \mu \mathrm{L}$ Counting Kit-8 (CCK-8) reagent was added to each well. After incubation for $1 \mathrm{~h}$, the absorbance value of cells in each well at $450 \mathrm{~nm}$ was detected by an automatic multifunctional enzyme plate analyzer.

\section{Colon formation test}

LNCaP cells and C4-2 cells were resuspended to adjust the cell concentration of $1 \times 10^{4}$ cells $/ \mathrm{mL}$. The culture dish was titled with agarose-Dulbecco's Modified Eagle Medium (DMEM), and cell suspension was added. A high concentration of agarose solution was added to the top of the dish. After the upper layer solidified, the cells were incubated in an incubator for 10 to 14 days, and the number of cell clones was observed under a microscope.

\section{Cell apoptosis assay}

LNCaP cells and C4-2 cells were prepared for cell suspension $\left(1 \times 10^{6}\right.$ cells $\left./ \mathrm{mL}\right)$. Five $\mu \mathrm{L}$ Annexin $\mathrm{V}$-fluorescein isothiocyanate (FITC) and $5 \mu \mathrm{L}$ PI were added and mixed 
gently. The cells were incubated at room temperature in the dark for $15 \mathrm{~min}$. The cell apoptosis rate was detected by flow cytometry.

\section{Cell migration and invasion assays}

A Transwell assay was performed to measure cell migration and invasion of PRAD cells. LNCaP cells and C4-2 cells were prepared for single-cell suspension with DMEM medium, and the cell concentration was adjusted to $1 \times 10^{5}$ cells $/ \mathrm{mL}$. The Transwell chamber was placed in a 24 -well plate. $800 \mu \mathrm{L}$ DMEM containing $10 \%$ FBS was added to the lower chamber. In the migration experiment, $200 \mu \mathrm{L}$ cell suspension $\left(1 \times 10^{4}\right.$ cells/well $)$ was added into the upper chamber. In the invasion experiment, Matrigel was added to the chamber before the cell was added. The 24-well plates were incubated in an incubator at $37{ }^{\circ} \mathrm{C}$ and $5 \% \mathrm{CO}_{2}$ for $24 \mathrm{~h}$. The Transwell chamber was cleaned with PBS, and then fixed with $4 \%$ paraformaldehyde for $20 \mathrm{~min}$, and stained with $0.5 \%$ crystal violet solution for $5 \mathrm{~min}$. The underlying cells were counted under a light microscope.

\section{Animal experiment}

BALB/C mice were purchased from Fuzhou Dingguo Biotechnology Co. Ltd. (Beijing, China). Male nude mice (aged 4-6 weeks with an average weight of 20 grams) were selected. The mice were kept in a constant temperature, humidity and sterile growth environment, and provided with sterile waster and sterile feed. LNCaP-shNC or LNCaP-shMAL2 cells $\left(2 \times 10^{6}\right.$ cells $\left./ \mathrm{ml}\right)$ were injected into mice ( $\mathrm{n}=5$ for each group) subcutaneously. Every 6 days, the tumors were removed to be photographed and weighted. The experiments were approved by the Animal Care and Use Committee of Beijing University of TCM (NO: A-2020-012). And animal experiments were conducted in compliance with institutional and national regulations for the care and use of laboratory animals.

\section{Statistical analysis}

SPSS 23.0 software was used to analyze data. Data were expressed as mean \pm standard deviation (SD). The Student's $t$-test was used to detect any differences between the groups. A $\mathrm{P}<0.05$ was considered statistically significant.

\section{Results}

\section{MAL2 was notably upregulated in PRAD tissues and cells}

To verify the potential role of MAL2 in PRAD, we searched the Gene Expression Profiling Interactive Analysis (GEPIA) database. MAL2 was more upregulated in PRAD tissues than non-tumor tissues (see Figure 1A). Next, we detected the expression level of MAL2 in 58 pairs of PRAD tissues and adjacent non-tumor tissues using a RT-qPCR assay and Western blot assay. As anticipated, the expression of MAL2 was remarkably higher in PRAD tissues than non-tumor tissues (see Figure 1B). Additionally, the protein expression of MAL2 was increased in 12 randomly selected PRAD tissues (see Figure 1C). Further, the protein expression of MAL2 was higher in certain PRAD cell lines (i.e., DU145, PC3, LNCaP, and C4-2) than RWPE-1 cell lines (see Figure 1D). Immunohistochemistry (IHC) staining confirmed that MAL2 was evidently more upregulated in PRAD tissues than corresponding adjacent non-tumor tissues (see Figure 1E). Thus, the results showed that MAL2 was mainly localized in the cytoplasm, and was in browngranular shape.

Based on the median value of MAL2, 58 PRAD patients were divided into high or low MAL2 expression groups. MAL2 expression was found to be closely related to TNM stage, the Gleason score and metastasis of PRAD patients (see Table 1). Further, a Kaplan Meier survival analysis showed that PRAD patients with a high expression of MAL2 had shorter survival time than PRAD patients with a low expression of MAL2 (see Figure $1 F$ ). Thus, MAL2 was found to be involved in the progression of PRAD, and associated with an unfavorable prognosis of PRAD.

\section{MAL2 silencing inbibited cell proliferation and induced cell apoptosis in PRAD}

The expression of MAL2 in LNCaP and C4-2 cells was higher than that in DU145 and PC3 cells (see Figure 1D). Thus, LNCaP and C4-2 cells were selected for the in vitro experiments. To explore the specific role of MAL2 in the progression of PRAD, sh-MAL2 was transfected into LNCaP and C4-2 cells. The expression of MAL2 was markedly decreased in $\mathrm{LNCaP}$ and $\mathrm{C} 4-2$ cells transfected with MAL2 knockdown (see Figure 2A). A CCK-8 assay and colon formation assay were used to measure the cell proliferation of LNCaP and C4-2 cells. Compared to 
A

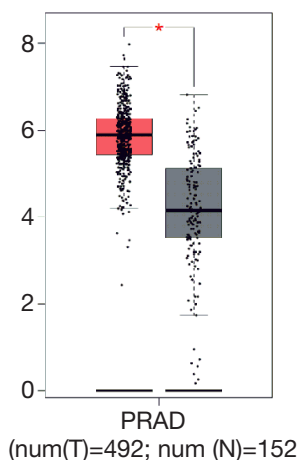

C

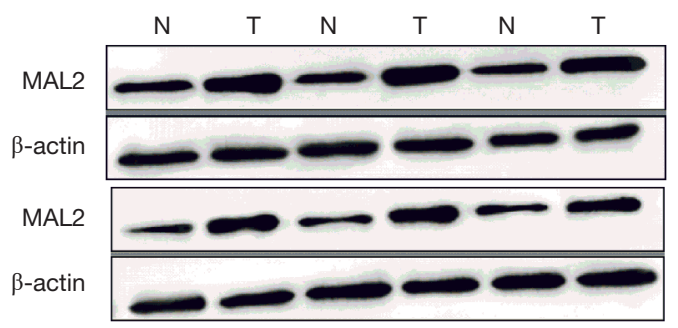

$E$

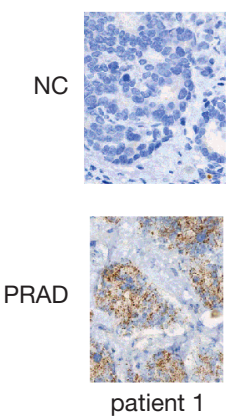

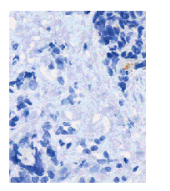

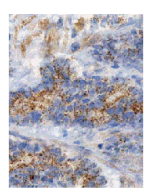

patient 2
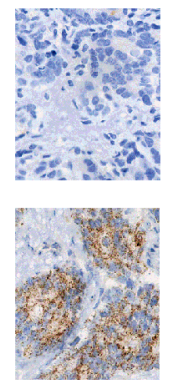

patient 3
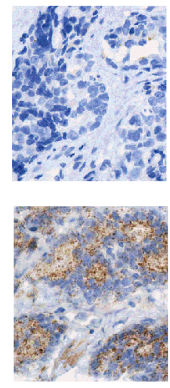

patient 4
B

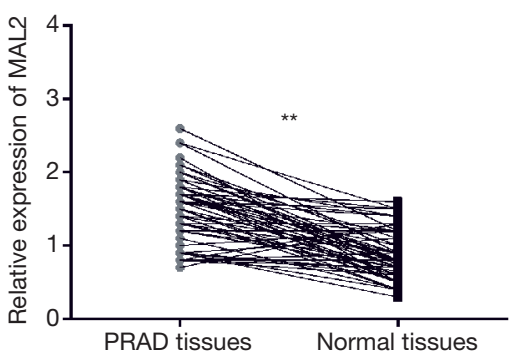

D

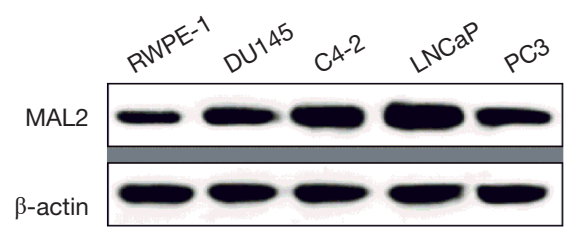

$\mathrm{F}$

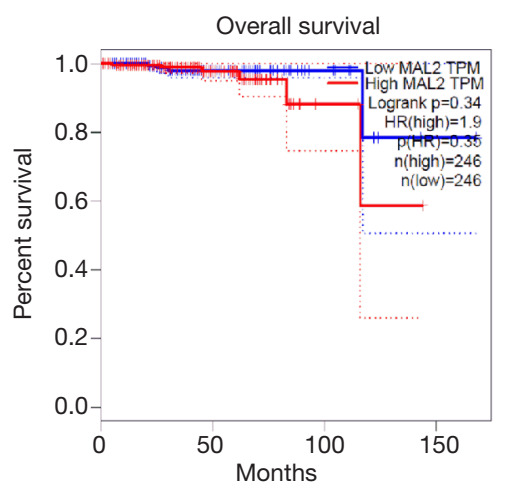

Figure 1 Myelin and lymphocyte protein 2 (MAL2) was notably upregulated in prostate adenocarcinoma (PRAD) tissues and cells. (A) The Gene Expression Profiling Interactive Analysis (GEPIA) database showed that MAL2 was more upregulated in PRAD tissues than non-tumor tissues; (B) MAL2 was overexpressed in 58 tissues of PRAD patients; (C) a Western blot assay showed that protein expression of MAL2 was upregulated in PRAD tissues (N: normal tissues, T: tumor tissues); (D) MAL2 was more highly upregulated in DU145, C42, LNCaP, and PC3 cells than RWPE-1 cells; (E) IHC staining showed the expression of MAL2 in PRAD patients (magnification: 40x); (F) the GEPIA database showed that the overall survival time of the high MAL2 expression group was shorter than that of the low MAL2 expression group. ${ }^{*} \mathrm{P}<0.05 ;{ }^{* *} \mathrm{P}<0.01$.

cells from the control group, the level of cell proliferation in LNCaP and C4-2 cells was obviously decreased (see Figure 2B,C). Similarly, the colon formation units were significantly reduced in $\mathrm{LNCaP}$ and $\mathrm{C} 4-2$ cells transfected with sh-MAL2 (see Figure 2D).

Next, a flow cytometry was performed to assess the cell apoptosis rates of $\mathrm{LNCaP}$ and $\mathrm{C} 4-2$ cells transfected with
sh-MAL2. As expected, cell apoptosis was higher in LNCaP and C4-2 cells transfected with sh-MAL2 than those of the control group (see Figure 2E). A Western blot assay was used to measure the expression of cell apoptosis-related proteins (i.e., Bcl-2, Bax, and cleaved caspase-3). MAL2 silencing dramatically decreased the expression of Bcl-2, while increasing the expression of Bax and cleaved caspase-3 
Table 1 Association between MAL2 expression and clinicopathological characteristics of patients with PRAD

\begin{tabular}{|c|c|c|c|c|c|}
\hline Characteristics & $\mathrm{n}=58$ & High expression $(n=30)$ & Low expression $(n=28)$ & $x^{2}$ value & $P$ value \\
\hline$<60$ years & 38 & 22 & 16 & & \\
\hline$\geq 60$ years & 20 & 8 & 12 & & \\
\hline PSA level & & & & 0.181 & 0.670 \\
\hline$>10$ & 42 & 21 & 21 & & \\
\hline TNM stage & & & & 5.524 & $0.019^{\star}$ \\
\hline$I+I I$ & 26 & 9 & 17 & & \\
\hline III + IV & 32 & 21 & 11 & & \\
\hline$>7$ & 19 & 14 & 5 & & \\
\hline Metastasis & & & & 4.392 & $0.036^{*}$ \\
\hline Present & 18 & 13 & 5 & & \\
\hline Absent & 40 & 17 & 23 & & \\
\hline
\end{tabular}

${ }^{*} \mathrm{P}<0.05$. PSA, prostate specific antigen; TNM, tumor node metastasis.

(see Figure 2F). All of the results suggested that MAL2 knockdown suppressed cell proliferation and promoted cell apoptosis in PRAD cells.

\section{MAL2 knockdown suppressed cell migration, invasion, and EMT progression in PRAD}

A Transwell assay was used to explore cell migration and the invasion ability of $\mathrm{LNCaP}$ and $\mathrm{C} 4-2$ cells. The results showed that cell migration was more blocked in $\mathrm{LNCaP}$ and C4-2 cells transfected with MAL2 knockdown than those of the control group (see Figure 3A). Similarly, cell invasion was significantly restrained by sh-MAL2 in LNCaP and C4-2 cells (see Figure 3B). A Western blot assay was used to detect expression levels of EMT related proteins. As Figure $3 C$ shows, the expression of E-cadherin was dramatically increased in $\mathrm{LNCaP}$ and C4-2 cells transfected with MAL2 knockdown, and the expression of $\mathrm{N}$-cadherin and Vimentin was decreased by sh-MAL2 (see Figure 3C). Thus, MAL2 knockdown was found to retard cell migration, invasion, and EMT progression in PRAD.

\section{MAL2 regulated PRAD progression through the Notch patbway}

To detect the special mechanism of MAL2 in PRAD progression, MAL2 overexpression or Notch inhibitorDAPT was transfected into LNCaP cells. A Western blot assay revealed that Notch-1 and Hes1 expression were obviously increased by MAL2 overexpression, but decreased by MAL2 + DAPT transfection (see Figure 4A). Next, cell proliferation was promoted by MAL2 overexpression, but inhibited by MAL2 + DAPT cotransfection (see Figure 4B,C). Further, cell migration and invasion were facilitated by MAL2 overexpression, but hampered by MAL2 + DAPT (see Figure 4D,E). In addition, DAPT was found to inhibit the effect of MAL2 on EMT progression in PRAD (see Figure 4F). Thus, MAL2 appears to regulate PRAD progression by modulating the Notch pathway.

\section{MAL2 silencing suppressed PRAD tumor growth in vivo}

To explore the effect of MAL2 in vivo, LNCaP cells 
A

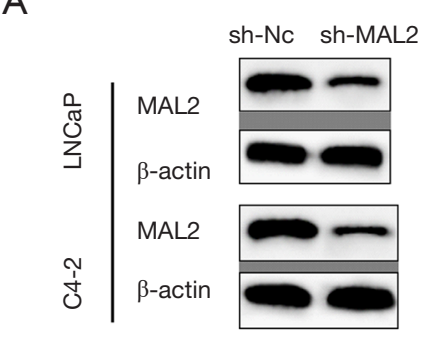

D

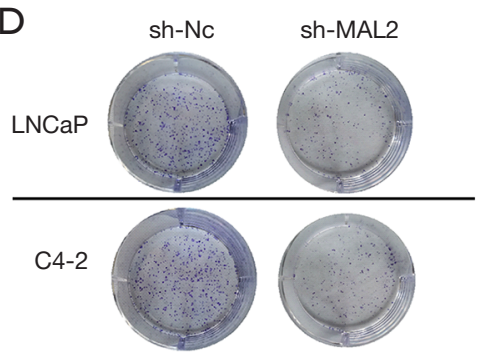

$E$

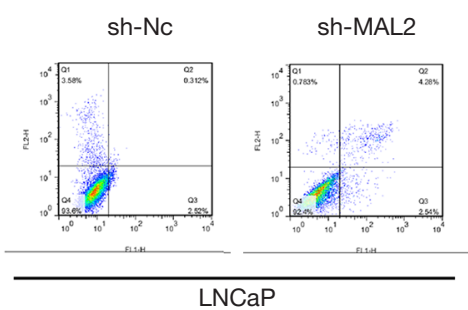

B
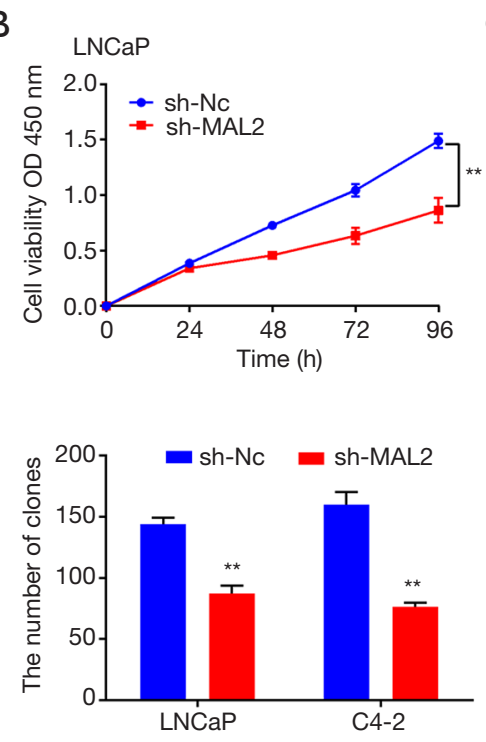

$\mathrm{F}$
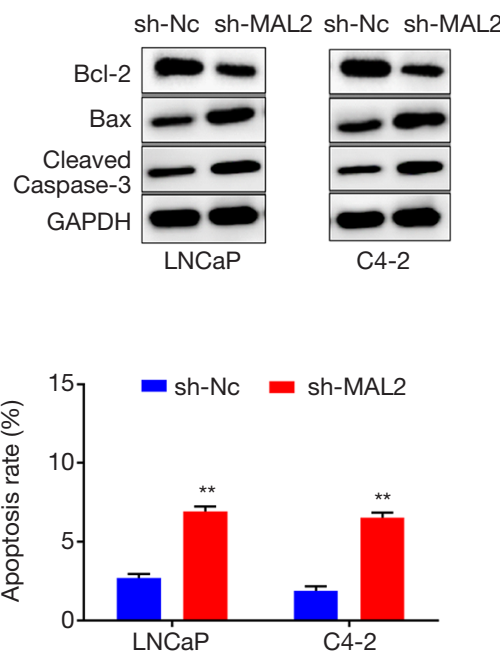

Figure 2 Myelin and lymphocyte protein 2 (MAL2) silencing inhibited cell proliferation and induced cell apoptosis in prostate adenocarcinoma (PRAD). (A) A Western blot assay showed that the expression of MAL2 was significantly reduced by sh-MAL2 in LNCaP and $\mathrm{C} 4-2$ cells; $(\mathrm{B}, \mathrm{C})$ the cell proliferation of $\mathrm{LNCaP}$ and $\mathrm{C} 4-2$ cells was significantly suppressed by sh-MAL2; (D) the number of colony in LNCaP and C4-2 cells was decreased by sh-MAL2; (E) MAL2 knockdown promoted cell apoptosis of LNCaP and C4-2 cells; (F) MAL2 knockdown dramatically decreased the expression of $\mathrm{Bcl}-2$, but increased the expression of Bax and cleaved caspase- $3 .{ }^{*} \mathrm{P}<0.01$.

transfected with sh-MAL2 or sh-NC were injected into the nude mice. Tumors were removed every 7 days for measurement. The results showed that the tumors removed from sh-MAL2 mice were obviously smaller than those removed from sh-NC mice (see Figure $5 A, B$ ). At 42 days after inoculation, the weight of the tumors from shMAL2 mice was lighter than that of the sh-NC mice (see Figure 5C). The results showed that MAL2 knockdown prevented PRAD progression in vivo.

\section{Discussion}

The incidence of PRAD has been increasing in recent years, and it has become the second major malignancy threating the health of men. As PRAD often has no obvious symptoms in the early stage, most PRAD patients are diagnosed at the middle or late stages (11). Thus, an indepth exploration of the molecules related to the occurrence and development of PRAD is of great significance for the early diagnosis and targeted therapy of PRAD. MAL2 is identified in proteins associated with membrane-binding events, and is an important component of the transcellular mechanism from the lateral wall of the base to the apex (12). Previous studies have shown that MAL2 is involved in the endocytosis of MHC-I complex in breast tumor cells, and reduces cytotoxicity by suppressing tumor antigen presentation (13). However, little is known about the effect of MAL2 in PRAD. The present study sought to verify the role of MAL2 in the progression of PRAD. The results showed that MAL2 was prominently overexpressed in the PRAD tissues of the tumor group compared to the non-tumor group. Further, PRAD patients with a high 
A

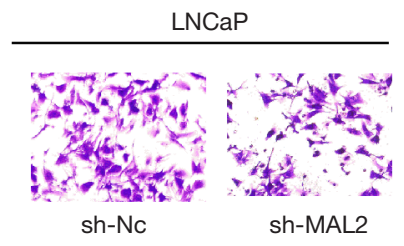

B

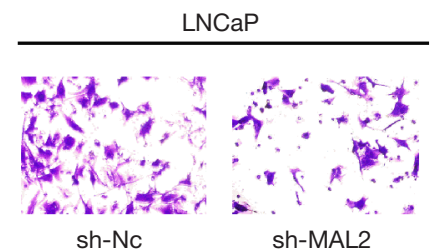

C

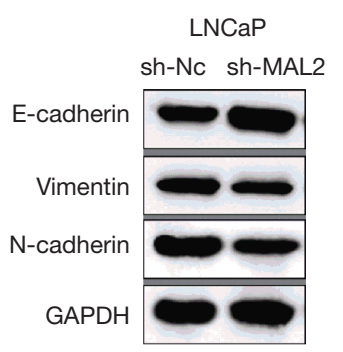

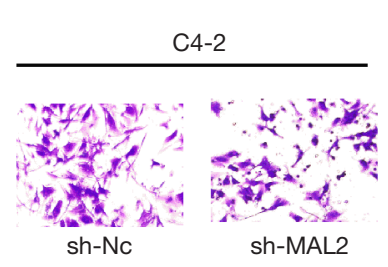

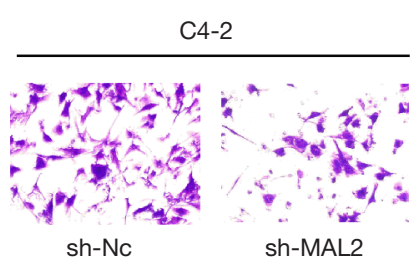

C4-2

sh-Nc sh-MAL2

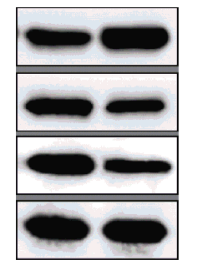

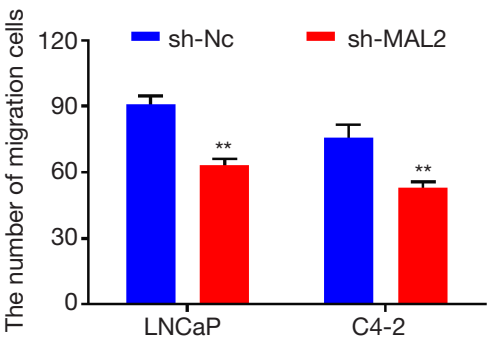
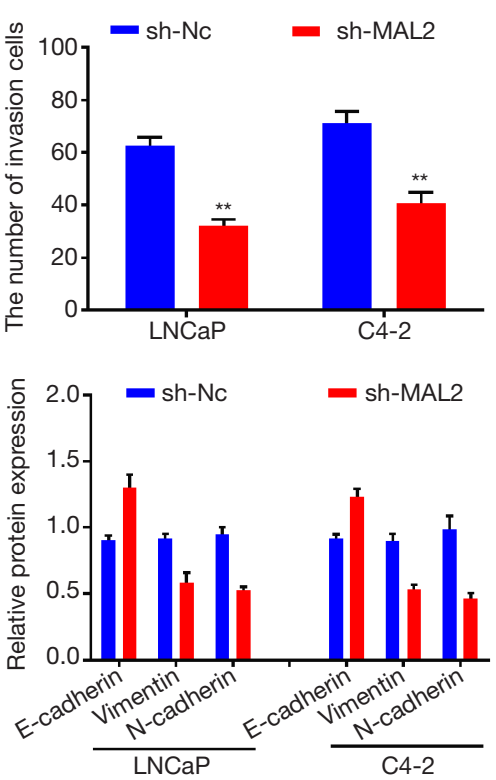

Figure 3 Myelin and lymphocyte protein 2 (MAL2) knockdown suppressed cell migration, invasion, and EMT progression in prostate adenocarcinoma (PRAD). (A) Cell migration was suppressed by MAL2 knockdown transfection in LNCaP and C4-2 cells (violet staining; scale bar =100 $\mathrm{m}$ ); (B) cell invasion was suppressed by MAL2 knockdown transfection in LNCaP and C4-2 cells (violet staining; scale bar $=100 \mu \mathrm{m}$ ); (C) MAL2 knockdown inhibited epithelial-mesenchymal transition (EMT) progression in LNCaP and C4-2 cells. ** $\mathrm{P}<0.01$.

expression of MAL2 had a shorter survival time than those with a low expression of MAL2. Consistent with our findings, MAL2 has also been found to be overexpressed in colorectal cancer, and identified as a prognostic factor for patients with colorectal cancer (14). The present study also showed that high MAL2 expression is closely related with TNM stage, the Gleason score and metastasis of PRAD patients.

To investigate how MAL2 affects the progression of PRAD, MAL2 knockdown was transfected into PRAD cells. The effect of MAL2 knockdown on cell growth, cell mobility, and the EMT progression of PRAD cells was then examined. As predicted, MAL2 knockdown obviously blocked cell proliferation, migration, invasion and EMT progression, and promoted the cell apoptosis of PRAD cells. Consistent with our findings, MAL2 deletion has been shown to block cell growth in breast cancer (BC), and MAL2 has been identified as a possible therapeutic target for BC immunotherapy (13). MAL2 has been found to be upregulated, and silence MAL2 suppressed cell proliferation and invasion in papillary thyroid carcinoma (15). Moreover, Shao et al. reported that the upregulation of RP11-284F21.9 facilitated oral squamous cell carcinoma progression by upregulating MAL2 expression (16). Renal cell carcinomas and $\mathrm{BC}$ have also been shown to have an extensive cytoplasmic distribution of MAL2 (17), which indicates that MAL2 might play a carcinogenic role in some cancers.

Notch signaling is a highly conserved signaling pathway that mediates the interaction between neighboring cells, and is widely found in various organisms (18). Studies have shown that the activation of the Notch signaling pathway plays a vital role in cell proliferation, differentiation and 
A

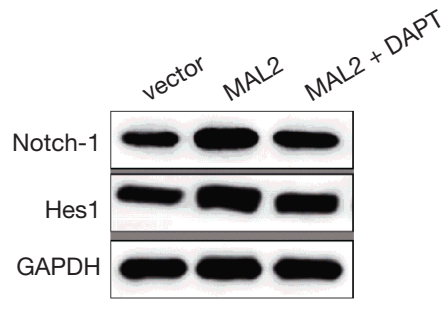

C
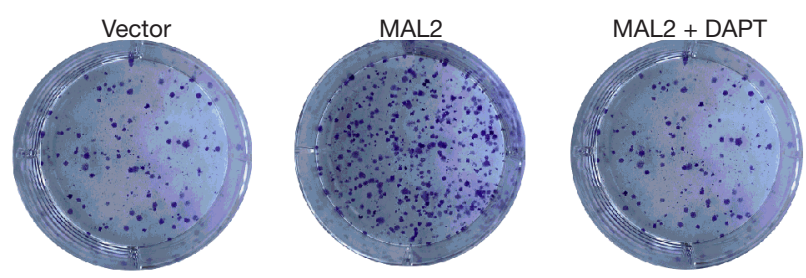

D
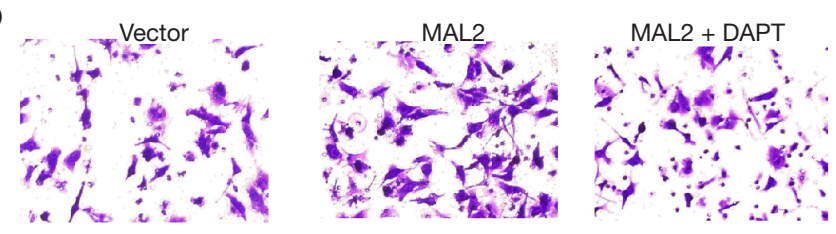

$\mathrm{E}$

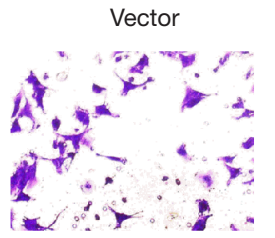

B
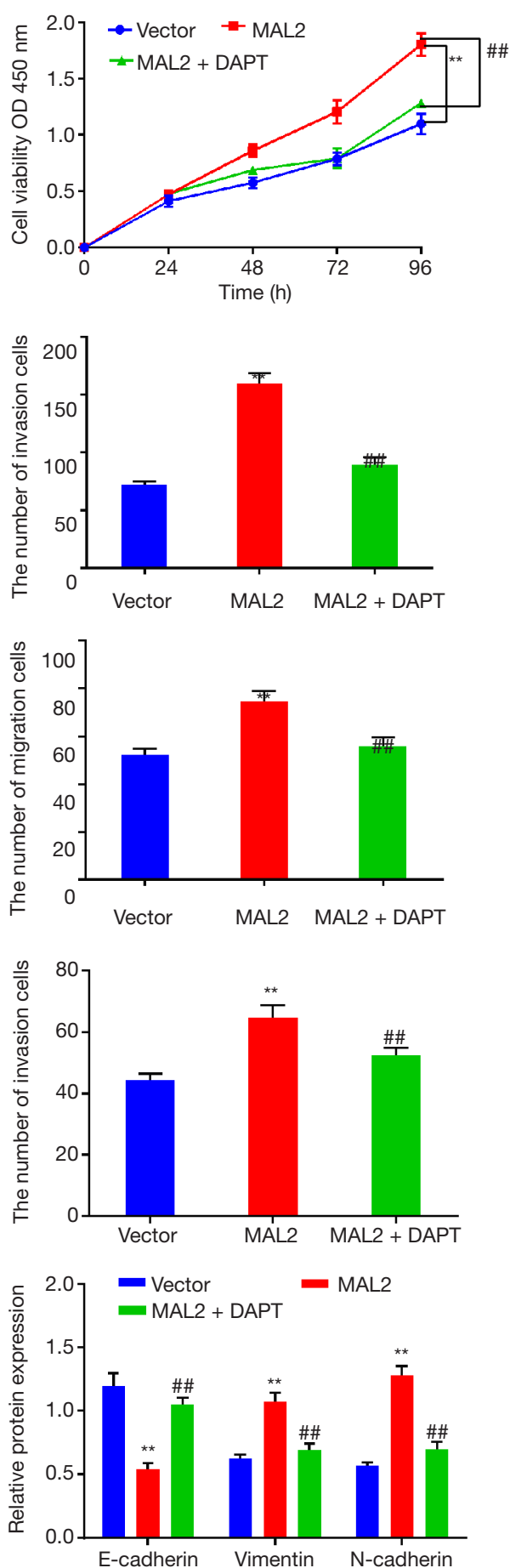

Figure 4 Myelin and lymphocyte protein 2 (MAL2) regulated prostate adenocarcinoma (PRAD) progression through the Notch pathway. (A) MAL2 increased the expression of Notch-1 and Hes1, while DAPT destroyed the effect of MAL2; (B,C) the cell proliferation of LNCaP cells was promoted by MAL2, but inhibited by MAL2 + DAPT co-transfection; (D) MAL2 significantly facilitated cell migration viability, while DAPT reversed the effect of MAL2 on LNCaP cell migration (violet staining; scale bar $=100 \mu \mathrm{m}$ ); (E) MAL2 significantly facilitated cell invasion viability, while DAPT reversed the effect of MAL2 on LNCaP cell invasion (violet staining; scale bar $=100 \mu \mathrm{m}$ ); (F) MAL2 significantly facilitated epithelial-mesenchymal transition (EMT) progression in LNCaP cells, while DAPT reversed the effect of MAL2 on

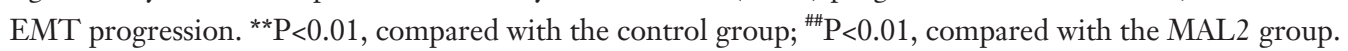


A

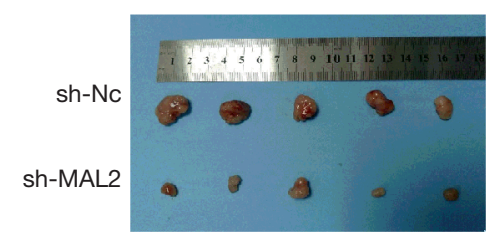

B

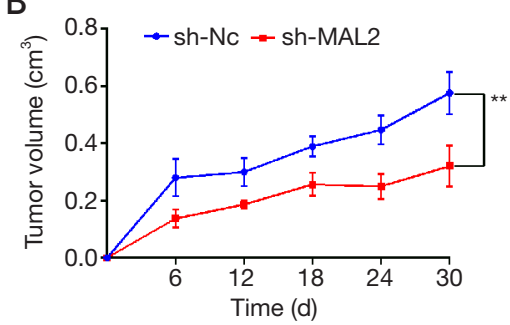

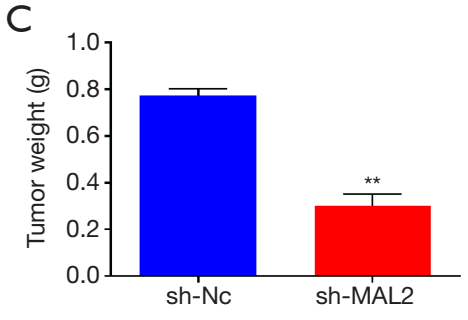

Figure 5 Myelin and lymphocyte protein 2 (MAL2) silencing suppressed prostate adenocarcinoma (PRAD) tumor growth in vivo. (A) The tumors removed from the sh-MAL2 mice were obviously smaller than those removed from sh-NC mice; (B) The tumor volumes were recorded every 6 days; (C) the average tumor weight was assessed at 30 days after cell injection. ${ }^{*}{ }^{*} \mathrm{P}<0.01$.

apoptosis (19-21). Recent findings have highlighted the role of the Notch signaling pathway in the development of a variety of human cancers, including BC, melanoma, ovarian cancer, and PRAD $(22,23)$. In the present study, the relationship between MAL2 and the Notch pathway was preliminarily studied in the progression of PRAD. The inhibitor of the Notch pathway was found to weaken the effect of MAL2 on cell progression in PRAD. Our data suggested that MAL2 regulates PRAD progression by modulating the Notch pathway.

\section{Conclusions}

In sum, our study showed that silencing MAL2 inhibited PRAD progression by regulating the Notch signaling pathway. In addition, it was suggested that MAL2 might be an effective target for the early diagnosis and treatment of PRAD. The results of this study have significant implications for the diagnosis and detection of PRAD.

\section{Acknowledgments}

Funding: The present study was supported by the National Natural Science Foundation Project (No. 7202115), and the Science and Technology Development Program of Traditional Chinese Medicine Bureau of Beijing (No. JJ2020-19).

\section{Footnote}

Reporting Checklist: The authors have completed the MDAR and ARRIVE reporting checklist. Available at http://dx.doi. org/10.21037/tau-21-244

Data Sharing Statement: Available at http://dx.doi. org/10.21037/tau-21-244

Conflicts of Interest: All authors have completed the ICMJE uniform disclosure form (available at http://dx.doi. org/10.21037/tau-21-244). The authors reported funding from the National Natural Science Foundation Project (No. 7202115), and from the Science and Technology Development Program of Traditional Chinese Medicine Bureau of Beijing (No. JJ2020-19). The authors have no other conflicts of interest to declare.

Ethical Statement: The authors are accountable for all aspects of the work in ensuring that questions related to the accuracy or integrity of any part of the work are appropriately investigated and resolved. All procedures performed in this study involving human participants were in accordance with the Declaration of Helsinki (as revised in 2013). The study was approved by the Ethics Committee of the Beijing University of Traditional Chinese Medicine (NO: 2019-028) and informed consent was taken from all the patients. The animal experiments were approved by the Animal Care and Use Committee of Beijing University of TCM (NO: A-2020-012). And animal experiments were conducted in compliance with institutional and national regulations for the care and use of laboratory animals.

Open Access Statement: This is an Open Access article distributed in accordance with the Creative Commons Attribution-NonCommercial-NoDerivs 4.0 International License (CC BY-NC-ND 4.0), which permits the noncommercial replication and distribution of the article with the strict proviso that no changes or edits are made and the original work is properly cited (including links to both the formal publication through the relevant DOI and the license). See: https://creativecommons.org/licenses/by-nc-nd/4.0/. 


\section{References}

1. Miller KD, Nogueira L, Mariotto A, et al. Cancer treatment and survivorship statistics, 2019. CA Cancer J Clin 2019;69:363-85.

2. Kimura T, Egawa S. Epidemiology of prostate cancer in Asian countries. INT J Urol 2018;25:524-31.

3. Bray F, Ferlay J, Soerjomataram I, et al. Global cancer statistics 2018: GLOBOCAN estimates of incidence and mortality worldwide for 36 cancers in 185 countries. CA Cancer J Clin 2018;68:394-424.

4. Serrell EC, Pitts D, Hayn M, et al. Review of the comparative effectiveness of radical prostatectomy, radiation therapy, or expectant management of localized prostate cancer in registry data. Urol Oncol 2018;36:183-92.

5. Fujizuka Y, Ito K, Oki R, et al. Predictive value of different prostate-specific antigen-based markers in men with baseline total prostate-specific antigen $<2.0 \mathrm{ng} / \mathrm{mL}$. Int J Urol 2017;24:602-9.

6. In JG, Striz A, Bernad A, et al. Serine/threonine kinase 16 and MAL2 regulate constitutive secretion of soluble cargo in hepatic cells. Biochem J 2014;463:201-13.

7. In JG, Tuma P. MAL2 selectively regulates polymeric IgA receptor delivery from the Golgi to the plasma membrane in WIF-B cells. Traffic 2010;11:1056-66.

8. Aranda JF, Reglero-Real N, Marcos-Ramiro B, et al. MYADM controls endothelial barrier function through ERM-dependent regulation of ICAM-1 expression. Mol Biol Cell 2013;24:483-94.

9. de Marco MC, Martín-Belmonte F, Kremer L, et al. MAL2, a novel raft protein of the MAL family, is an essential component of the machinery for transcytosis in hepatoma HepG2 cells. J Cell Biol 2002;159:37-44.

10. Eguchi D, Ohuchida K, Kozono S, et al. MAL2 expression predicts distant metastasis and short survival in pancreatic cancer. Surgery 2013;154:573-82.

11. Dimakakos A, Armakolas A, Koutsilieris M. Novel tools for prostate cancer prognosis, diagnosis, and follow-up. Biomed Res Int 2014;2014:890697.

Cite this article as: Zheng C, Wang J, Zhang J, Hou S, Zheng Y, Wang Q. Myelin and lymphocyte protein 2 regulates cell proliferation and metastasis through the Notch pathway in prostate adenocarcinoma. Transl Androl Urol 2021;10(5):20672077. doi: 10.21037/tau-21-244
12. Wilson SH, Bailey A, Nourse C, et al. Identification of MAL2, a novel member of the mal proteolipid family, though interactions with TPD52-like proteins in the yeast two-hybrid system. Genomics 2001;76:81-8.

13. Fang Y, Wang L, Wan C, et al. MAL2 drives immune evasion in breast cancer by suppressing tumor antigen presentation. J Clin Invest 2021;131:e140837.

14. Li J, Li Y, Liu H, et al. The four-transmembrane protein MAL2 and tumor protein D52 (TPD52) are highly expressed in colorectal cancer and correlated with poor prognosis. PLoS One 2017;12:e0178515.

15. Gao X, Chen Z, Li A, et al. MiR-129 regulates growth and invasion by targeting MAL2 in papillary thyroid carcinoma. Biomed Pharmacother 2018, 105:1072-1078.

16. Shao B, Fu X, Li X, et al. RP11-284F21.9 promotes oral squamous cell carcinoma development via the miR-3835p/MAL2 axis. J Oral Pathol Med 2020;49:21-9.

17. Fanayan S, Shehata M, Agterof A, et al. Mucin 1 (MUC1) is a novel partner for MAL2 in breast carcinoma cells. Bmc Cell Biol 2009;10:7.

18. Bray SJ. Notch signalling: a simple pathway becomes complex. Nat Rev Mol Cell Biol 2006;7:678-89.

19. Monticone G, Miele L. Notch Pathway: A Journey from Notching Phenotypes to Cancer Immunotherapy. Adv Exp Med Biol 2021;1287:201-22.

20. Reiff T, Baeumers M, Tibbe C, et al. Unravelling of Hidden Secrets: The Tumour Suppressor Lethal (2) Giant Discs (Lgd)/CC2D1, Notch Signalling and Cancer. Adv Exp Med Biol 2021;1287:31-46.

21. Misiorek JO, Przybyszewska-Podstawka A, Kałafut J, et al. Context Matters: NOTCH Signatures and Pathway in Cancer Progression and Metastasis. Cells 2021;10:94.

22. Fasoulakis Z, Daskalakis G, Theodora M, et al. The Relevance of Notch Signaling in Cancer Progression. Adv Exp Med Biol 2021;1287:169-81.

23. Deng G, Ma L, Meng Q, et al. Notch signaling in the prostate: critical roles during development and in the hallmarks of prostate cancer biology. J Cancer Res Clin Oncol 2016;142:531-47. 